\title{
In situ imaging combined with deep learning for crystallization process monitoring: Application to cephalexin production
}

Hossein Salami ${ }^{1},{ }^{*}$, Matthew A. McDonald ${ }^{1}$, Andreas S. Bommarius ${ }^{1}$, Ronald W. Rousseau ${ }^{1}$, Martha A. Grover $^{1}$

${ }^{1}$ School of Chemical \& Biomolecular Engineering, Georgia Institute of Technology, Atlanta GA 30332

\section{Supplementary Information}

\section{Cephalexin and phenylglycine solubility as a function of $\mathrm{pH}$}

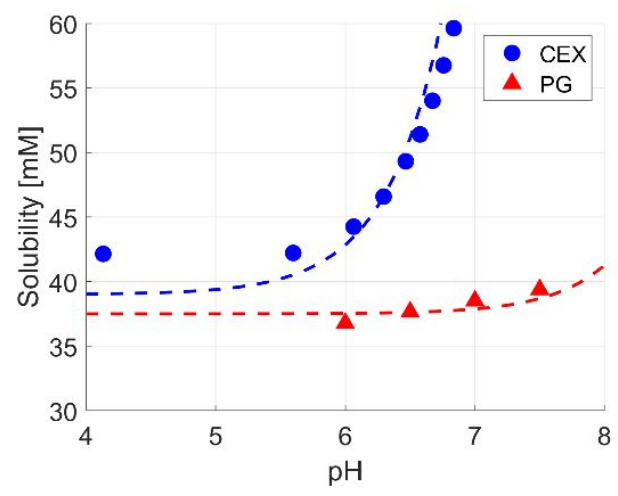

Figure S1. Solubility of cephalexin and phenylglycine in water as a function of $\mathrm{pH}$. Cephalexin data are from McDonald et al., 2019 [1], phenylglycine data are from Santana et al., 2010 [2]. Dotted lines are obtained by fitting to the data the equation $\mathrm{c}^{*}=\mathrm{c}^{*} \mathrm{ip}\left(1+10^{(-\mathrm{pKa}+\mathrm{pH})}\right)$ where $\mathrm{c}^{*}$ is the solubility, pKa is the acid dissociation constant, and $\mathrm{c}^{*}$ ip is the solubility at the isoelectric point and is the fitted parameter (Ref. 31 of the main article).

\section{Estimation of supersaturation generation during PG crystallization}

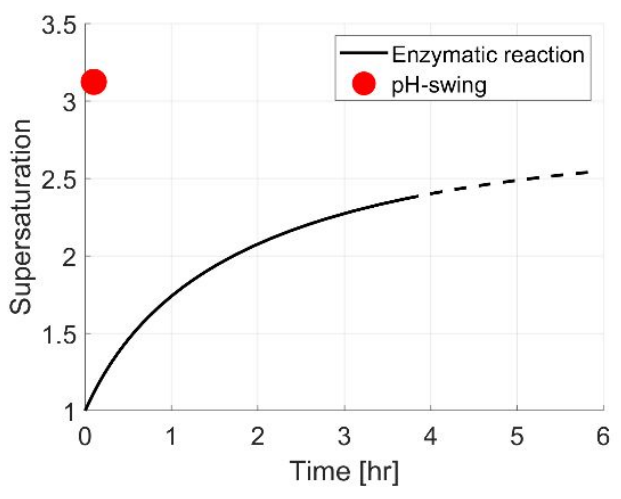

Figure S2. Estimation of supersaturation generation for two cases of producing phenylglycine particles, $\mathrm{pH}$ swing, and enzymatic synthesis. The curve for enzymatic synthesis is produced using the enzyme kinetic parameters reported by McDonald et al. (Ref. 31 of the main article). The dotted portion of the curve represents the potential start of primary nucleation. 


\section{ResNet-50 performance}
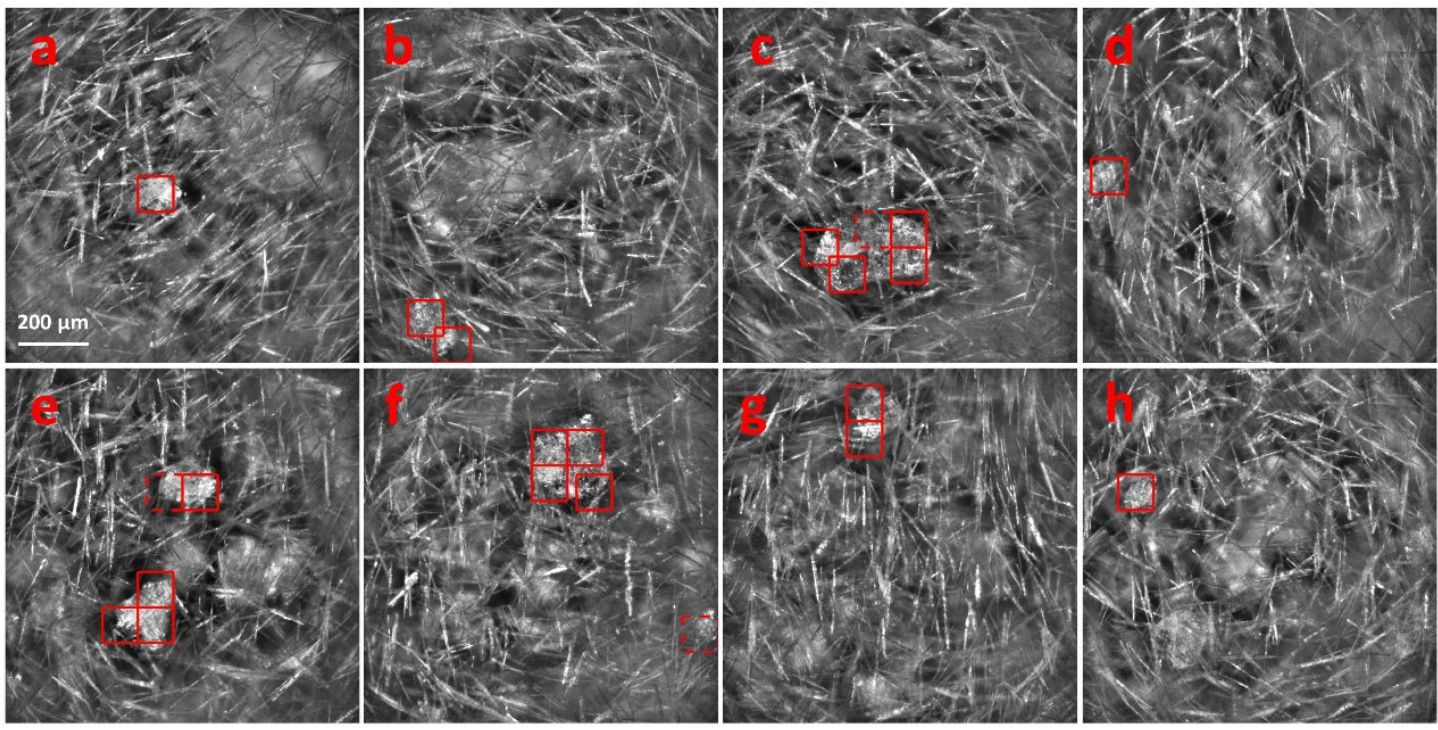

Figure S3. Results of applying the image analysis model (with ResNet-50 CNN) to a series of in situ images acquired from a crystal slurry with added $\mathrm{pH}$-made $\mathrm{PG}$ crystals. Solid red boxes correspond to regions that are decided to have a PG particle based on the classification threshold of 0.9. Dashed boxes are additionally flagged regions based on the classification threshold of 0.8 .

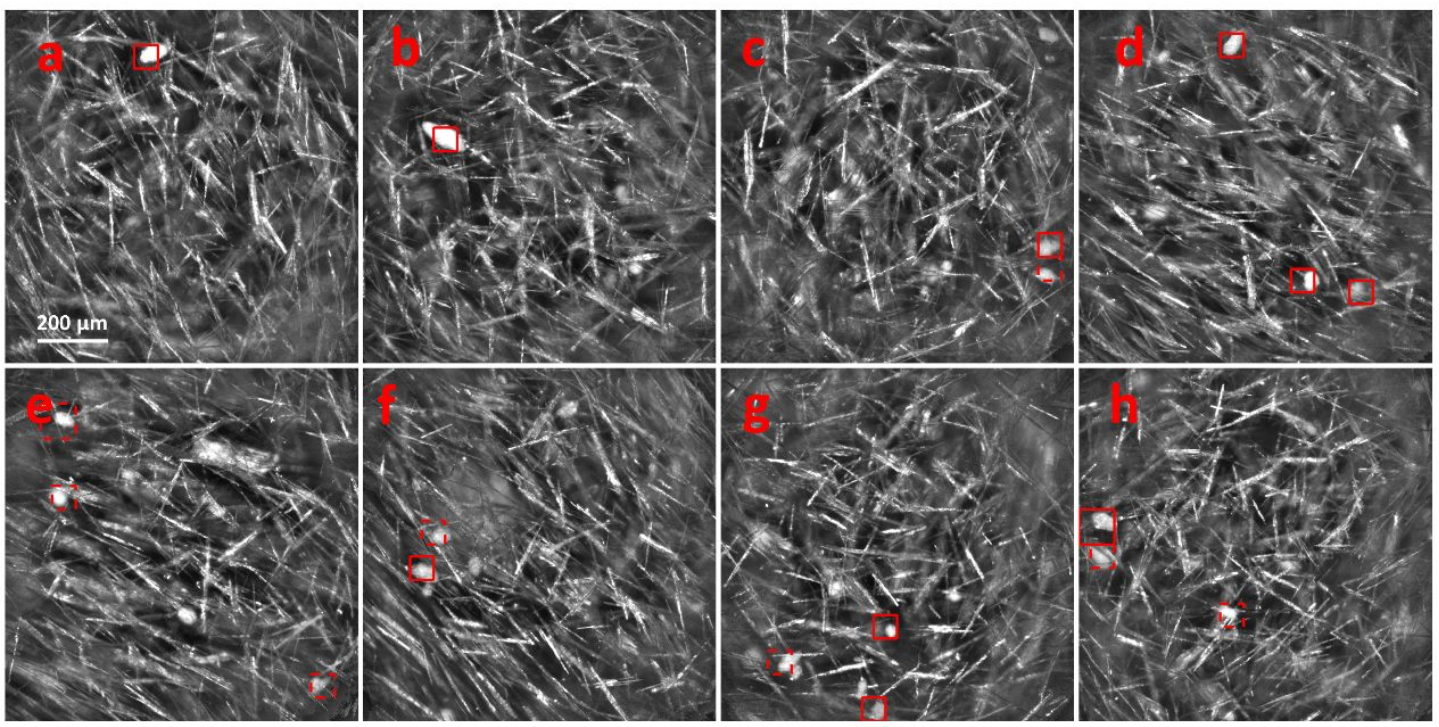

Figure S4. Results of applying the image analysis model (with ResNet-50 CNN) to a series of in situ images acquired from a crystal slurry with added enzymatically-made PG particles. Solid red boxes correspond to regions that are decided to have a PG particle based on the classification threshold of 0.9. Dashed boxes are additionally flagged regions based on the classification threshold of 0.8 . 


\section{Parameters used for the estimation of PG particles number density}

Total slurry volume $=65 \mathrm{~mL} \quad$ Added PG batches $=0.05 \mathrm{gr}$ at a time

PG solid density $\sim 1.2 \mathrm{~g} / \mathrm{mL} \quad P G$ shape factor $\sim 0.3$ (average of tetrahedron and octahedron geometries [3])

\section{Structure of ResNet-18 and ResNet-50}

ResNet-18

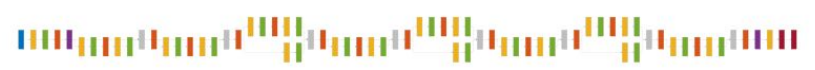

ResNet-50

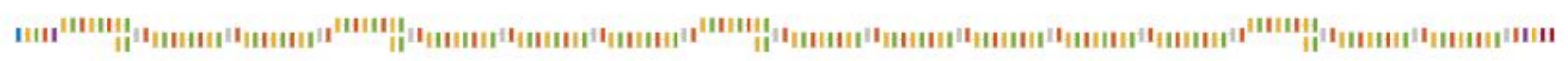

Blue blocks correspond to the image input layer, yellow to convolution filters, green to batch normalization, red to ReLU activation, violet to max pooling. The two last layers in each network are the fully connected layer and the accompanying classification layer. Structures are drawn via MATLAB 2020b deepNetworkDesigner app.

\section{Derivation of Eqn. (2)}

Assuming an ideal observer, the probability of a PG particle in the vessel not showing up in the volume in front of the PVM probe window is $1-\frac{V_{\text {sampling box }}}{V_{\text {vessel }}}$. For multiple, $N_{P G}$, particles this becomes $\left(1-\frac{V_{\text {sampling box }}}{V_{\text {vessel }}}\right)^{N_{P G}}$ which is the probability that no PG particle is placed in the volume in front of the PVM probe. Therefore, $1-\left(1-\frac{V_{\text {sampling box }}}{V_{\text {vessel }}}\right)^{N_{P G}}$ is the probability that at least one PG particle exists in the volume in front of the PVM probe (leading to its detection and the captured image being flagged).

\section{References}

[1] McDonald, M.A., Marshall, G.D., Bommarius, A.S., Grover, M.A. and Rousseau, R.W., 2019. Crystallization kinetics of cephalexin monohydrate in the presence of cephalexin precursors. Crystal Growth \& Design, 19(9), pp.5065-5074.

[2] Santana, M., Ribeiro, M.P., Leite, G.A., Giordano, R.L., Giordano, R.C. and Mattedi, S., 2010. Solidliquid equilibrium of substrates and products of the enzymatic synthesis of ampicillin. AIChE journal, 56(6), pp.1578-1583.

[3] Chianese, A., 2012. Characterization of crystal size distribution. Industrial Crystallization Process Monitoring and Control, pp.1-6. 